tion for Chapters 5-7. These are written in terms of the utmost gonerality, with no examples, and contain a numbor of sentences at the exact meaning of which I can only guess.

I noticed errors in two formulae. In the equation at the foot of page 69 the term $p_{0}^{n} /(n-1)$ should be $p_{0}^{n} / n$, and (more seriously) in the formula for $f$ on page 82 the complete numerator of the fraction should be, but is not, squared.

The book is completed by four chapters on sequential sampling, lot acceptance sampling plans (illustrated by discussion of two U.S. Defense Department Military Standards), sample-size precision schedules, and decision functions, a threo-page bibliography, and a useful 28-pige appendix of tables. The tables are photographically reproduced, with results that though always legible are sometimes unattractive: this detracts from the otherwise high standard of production.

Perhaps this book can best be summed up as a cook-book with pretensions: as such it is expensive at the price.

\section{R. SAMPFORD}

\section{General Protozoology}

By V. A. Dogiel. Revised by J. I. Poljanskij and E. M. Chejsin. Second edition. Pp. xiv + 747. (Oxford: Clarendon Press; London: Oxford University Press, 1965.) 147s. net.

A NEW text-book devoted to the general aspects of protozoology has been greatly needed, as the classical books of the past (Doflein, Wenyon, Calkins, etc.) have become much out of date. Included in the latter was the General Protozoology of Dogiel, published in 1951 in Russian. It has now been practically rewritten by two of his students, J. I. Poljanskij and E. M. Chejsin, from the Institute of Cytology, Leningrad, and well translated into English by C. A. Hoare. It will prove invaluable to protozoologists and biologists in general, on account of the lucid exposition of the main features of the protozoa, with particular reference to their morphology, cell-division and evolution. Chapters are accordingly devoted to the cytoplasm, nucleus, skeletal and fibrillar structures, organelles of locomotion, methods of reproduction and regeneration, and evolution. The physiology of the organisms, including metabolism and irritability, are considered separately, while there are two special chapters on life-cycles and ecology. Examples are taken from the free-living and parasitic protozoa, but there is, of course, no detailed treatment of individual species, and medical and veterinary protozoology, as such, is entirely omitted from consideration. This book provides the fundamental background to a subject which is advancing so rapidly, particularly in the elucidation of fine structure, genetics and metabolism, that still another edition will be required in a fow years' time. Readers will find it stimulating and challenging; in many places, it is emphasized that the "problem leaves much to be investigated" and this recommendation applies as much to fundamental phenomena of biology as to host-parasite relationships, systematics and other questions of more specialized interest. The book is illustrated with 326 figures of exceptional clarity and its referenees are thoroughly international.

$$
\text { P. C. C. Garnham }
$$

\section{Biophysics and Cybernetic Systems}

Edited by Myles Maxfield, Arthur Callahan and Lawrence J. Fogel. (Proceodings of the Second Cybernetic Sciences Symposium, October 13, 1964.) Pp. vii + 184. (Washington, D.C.: Spartan Books, Inc.; London: Macmillan and Co., Ltd., 1965.) 45s. net.
INTENDING readers would be well advised to begin with this book at the Appendix. The conference of Californian scientists which it reports is one of the many crossdisciplinary explorations that seem nowadays to find eager publishers, and the Appendix contains a candid and not altogether flattering debate as to its value. The time must surely come soon when "quality control" will be applied to papers for symposium volumes as for those in reputable journals, and publishers who neglect to do so will bocome known accordingly.

It is not suggested that all or even most of the papers here presented fall short by journal standards. The subjects range from the biophysics of natural and synthetic membranes to the modelling of complex biological processes and the theory of artificial intelligence, and almost all the material will be of some interest to specialists in the respective fields. Most original and stimulating, porhaps, is L. J. Fogol's discussion of "Artificial Intelligence through a Simulation of Evolution". He proposes a method of allowing an automaton to evolve its own logic by a search process of "natural selection" which he considers to have reasonable prospects of convergence. In a different direction R. L. Binggeli's finding, that pigeons improve in left-right discrimination whon they lose one optic tectum, throws intriguing light on the functional significance of brain symmetry.

But a good many of the roports here presented-espocially in the field of network-modelling-are acknowledged to be preliminary or inconclusive. In a research meeting of specialists this may be positively beneficial to the freedom and vigour of discussion. Whether it justifies their addition in that form to world literature is another question.

D. M. MACKAY

\section{Vistas in Astronomy}

Edited by Arthur Boer. Vol. 6: Celestial Mechanies, Instrumentation, Meridian Astronomy, Solar Research, Astrophysics. Pp. vii +214 . (London and New York: Pergamon Press, 1965.) 84s. net.

THIs is the sixth volume in a series containing sets of review papers on subjects covering all the fields of astronomy and astrophysies, written by internationally recognized specialists. The volume includes seven such papers by eight scientists, working in six different countries.

Of particular interest is the paper on relativistic theories of gravitation by G. J. Whitrow and G. E. Morduch, in which one finds an extensive comparison of the effects predicted by the general relativity and eight Lorentzinvariant theories of gravitation for the three astronomical tests of relativity: the deflexion of a light-ray near a mass; the gravitational red-shift; and the advance of porihelion in the two-body problem. Additional advances of perihelion and node due to the rotation of the central body are also considered. None of the theories are strictly equivalent to the general relativity in their predicted effects. The final conclusions are particularly well presented and can be read fruitfully without going through all the mathematics.

Another interesting article is that on the diffuse emission nebulae by S. R. Pottasch. The problems of structure, brightness and interpretation of thoir spectra are very clearly presented in a broad review of all data concerning these important members of our Galaxy.

Tho other papers aro: one on catalogues of meridian observations of stars (F. Schmeidler); two on optical and soeing problems of long-focus refractors (P. A. Ianna and S. L. Lippincott); and two on active regions of the Sun (S. B. Pikelner and J. Kleczek). All, without doubt, will bo quoted frequently in future works and constitute an important contribution in their respective fields.

J. Kovalevsky 